\title{
Local Information from Range-Speed Radar Sequences
}

\author{
Ph. van Dorp ${ }^{1}$, F.C.A. Groen ${ }^{2}$ \\ ${ }^{1}$ Department of Radar and Electronic Warfare, \\ TNO Defence Security and Safety, \\ P.O. Box 96864, 2509 JG The Hague, The Netherlands \\ Phone: +31 70 3740000, Fax: +31 70 3740654, E-mail: philip.vandorp@tno.nl. \\ ${ }^{2}$ Intelligent Autonomous System (IAS) Group \\ University of Amsterdam, \\ Kruislaan 403, 1098 SJ Amsterdam, The Netherlands \\ Phone: +31 20 5257461, Fax: +31 20 5257490, E-mail: groen@ science.uva.nl.
}

\begin{abstract}
Radar observes targets, but they remain difficult to interpret due to the difficulty in analysing the radar range-speed sequences. The need for accurate analyses tools increases in case of extended target behaviour or multiple channel radars which give additional observation angles. Extended targets are targets with multiple scatterer responses which disturb each other and give a blurred target response. We investigate here the approach of deconvoluting the range-speed response with a point spread function and interpolate the range-speed positions to get the inner structure of the extended target. The deconvolution gives the individual elements of the extended target. The range-speed interpolation gives accurate position information. The positions and additional observation angle information are tracked with a filter. We demonstrate the approach with real radar measurements.
\end{abstract}

Keywords - radar signal processing, deconvolution, interpolation, tracking

\section{INTRODUCTION}

Radars observe targets in time. Moving targets composed of individual moving elements give specific modulation characteristics in the range-speed response or space with amplitude and phase. These characteristics vary in time and this time behaviour depends on the motion of the individual elements of the target. A human target has moving body elements which have specific relations. Estimation of the kinetics of moving body elements is the topic of this study.

If the object or a part of the object has rotation or translation in addition to the body motion, it might induce a frequency modulation and amplitude modulation on the backscattered radar signal. These modulations give sidebands on both sides of the body component in the spectrum. This micro-Doppler or micro-motion effect is observed in the 2-D speed-time space, see [3]. If the micro-motion has a range dependency the range information is added, this is the 3-D range-speed-time space referred as the 3-D cubic micro-motion feature space, see [17],
[12] and [10]. A theoretical study of micro-motion of simple objects like cubes and rods is given by Chen [3] and Mitra [13]. Chen derived a mathematical framework solving the micro-motion modulations induced by vibration, rotation, tumbling and coning motions and applied to point scatterers target models.

Another approach is the feature-based approach that estimates the parameters of a human walking model. Model-based approaches with the Boulic model for estimating human motion parameters are described in [4], [5] and [6].

In this paper we modelled the extended target as a sum of individual moving point scatterers in space. Examples of extended point targets are walking humans, flapping birds and cyclists. These point scatterers induce peaks in the rangespeed response. Estimation of these peaks in the range-speed response and observation angles gives the kinematics of the point scatterers. The point scatterers trajectories will be used to identify the scattering points of the target.

Range-speed-time measurements are complex values with amplitude and phase both varying in time. The problem is to find how the amplitude and phase evolve in time in the rangespeed response. Fleet [7] [8] presents component velocity from image sequences based onto the complex output velocity filters. Sandwell [14] use the phase gradient approach to stacking interferograms. Radar phase information is very sensitive to noise and multipath with other reflection points and we limited the study to range-speed amplitude information.

Range resolution depends on the bandwidth of the transmitted signal of the used Frequency Modulated Continuous Radar (FMCW) radar. Government regulation limits a higher bandwidth and it requires extra costs due to expensive hardware and more processing capacity. Higher range resolution gives more details of the observed object. One method to improve the range resolution is sharping the prominent peaks in range-speed response with the Quadratically Interpolated FFT method, see [16] and generate an improved range-speed response. 
The method we present gives the local amplitude information of the range-speed sequence combined with interferometric observation angle in the vertical and horizontal dimension. In order to perform interferometric radar angle estimation at least two receiver channels are required (more are preferred) for each dimension. The local amplitude information is estimated by deconvolution of the range-speed response with a point spread function. The range-speed peak positions are calculated with range-speed interpolation. The interferometric phase angle provides the observation angle. Tracking the peak positions and observation angles gives the time evolution.

The paper is organized as follows: Sections 2 gives the radar signal processing. The approach is implemented and demonstrations of the technique are given in section 3 . These demonstrations involve real radar measurements. Finally, section 4 summarizes the paper, and draw conclusions from it.

\section{RADAR SIGNAL PROCESSING}

The radar signal processing generates the range-speed response from the received signal. These signals form the basis of the processing. Two independent dual channel interferometric radar are used. One radar measures in the vertical plane the other radar in the horizontal plane. Each dual channel interferometric radar has two range-speed responses, see [15].

The processing steps are:

- Compute the range-speed response of all four receiver channels.

- Calculate the vertical and horizontal observation angles.

- Deconvolution of the vertical and horizontal range-speed responses with the point spread function

- Apply the Quadratically Interpolated FFT method onto each peak of the horizontal and vertical range-speed response.

- Calculate the projections of the range-speed response.

- Kalman tracking of the peaks.

A. Range-speed processing

A dual channel interferometric radar gives the complex range-speed responses $R_{1}(r, v, t)$ and $R_{2}(r, v, t)$, see [15]. In order to reduce noise and interfering signal distortion the range-speed response is smoothed over the time window $T$ :

$$
R_{v}(r, v, t)=\sum_{t-T / 2}^{t+T / 2} \frac{1}{2}\left(\left|R_{1}(r, v, t)\right|+\left|\left(R_{2}(r, v, t)\right)\right|\right)
$$

The time window $T$ depends on the kinematics of the targets. The observation angle is calculated with the coherence or interferometric correlation between the two signals, see [9]:

$$
\begin{aligned}
& C_{v}(r, v, t)= \\
& \frac{\sum_{\text {region } r, v, t} R_{1}(r, v, t) \operatorname{conj}\left(R_{2}(r, v, t)\right)}{\sqrt{\sum_{\text {region } r, v, t}\left|R_{1}(r, v, t)\right|^{2} \sum_{\text {region } r, v, t}\left|R_{2}(r, v, t)\right|^{2}}}
\end{aligned}
$$

The $C_{v}(r, v, t)$ smoothing region is over the time window and a range-speed window. The range-speed window is related with the range-speed extension of a point scatterer. This extension is greater than one element, the eight neighbour elements is a minimum choice. The coherence is a complex value, the angle $\angle C(r, v, t)$ is the interferometric phase and the absolute value $|C(r, v, t)|$ indicates the multipath. If there is no multipath the absolute coherence is one and with multipath the coherence is smaller than one. Radar multipath echoes from an actual target causes ghost targets to appear. Multipath may also originate from multipath echoes from valid targets due to ground reflection. An additional constraint is the target amplitude: small amplitudes indicate noise with unpredictable observation angle. The interferometric phase gives the observation angle of the point target:

$$
\alpha_{v}(r, v, t)=\sin ^{-1}\left(\angle C_{v}(r, v, t) \lambda 2 \pi d\right)
$$

where $\lambda$ is the centre wavelength of the radar and $d$ the distance between the two receiver channels. The horizontal radar processing is equivalent to the vertical processing with rangespeed response $R_{h}(r, v, t)$ and observation angle $\alpha_{h}(r, v, t)$.

\section{$B$. Range-Speed deconvolution}

The restoration is performed by reversing the convolution operator inherent in the radar system. If the radar system is modelled as a convolution of the "true range-speed" response $\hat{R}_{v}(r, v)$ with the point spread function $P S F(r, v)$, then a deconvolution of the raw range-speed response should restore the true range-speed response. The purpose of deconvolution is to reveal the inner structure of an extended target. The point spread function is the response of a simulated point target with constant speed. The point spread function is non-negative symmetric in range and speed and its absolute values are normalized to one: $\sum P S F(r, v)=1$. The deconvolution is applied to the absolute value of the range-speed response $R_{v}(r, v, t)$ :

$$
R_{v}(r, v, t)=\hat{R}_{v}(r, v) * P S F(r, v, t)+N(r, v, t)
$$

where $N(r, v)$ is additive noise. The Lucy-Richardson deconvolution method [11] is applied to solve the inverse problem:

$$
\begin{aligned}
& \min \left(J\left(Q_{v}(r, v, t)\right)=\right. \\
& \quad \min \left(\left|R_{v}(r, v, t)-\hat{R}_{v}(r, v) * \operatorname{PSF}(r, v, t)\right|^{2}\right)
\end{aligned}
$$

\section{Quadratic Peak Interpolation}

The range-speed peak positions in true range-speed responses are estimated with quadratic peak interpolation [1]. A peak is a $\hat{R}_{v}(r, v, t)$ position were all eight neighbours lay below the peak value. The peak interpolation in range and speed are equivalent. Let $k_{0}$ the bin number of the maximum peak and $u_{k_{0}}$ the log magnitude at the bin and $u_{k_{0}-1}$ and $u_{k_{0}+1}$ be the two neighbours of it. The log-magnitude peaks are interpolated as: 


$$
u(k)=a\left(k-k_{0}\right)^{2}+b\left(k-k_{0}\right)+c
$$

and:

$$
\begin{gathered}
a=\left(u_{k_{0}+1}-2 u_{k_{0}}+u_{k_{0}-1}\right) / 2 \\
b=\left(u_{k_{0}+1}-u_{k_{0}-1}\right) / 2 \\
c=u_{k_{0}}
\end{gathered}
$$

The position of the maximum is $\Delta=-b /(2 a)$ with magnitude $u(\Delta)=a \Delta^{2}+b \Delta+c$. The total peak amplitude is the average value of the interpolated range position and the interpolated speed position. The angle is the average of a window around the peak position.

\section{Parameter Modifications}

The synthesis of the range-speed response $\check{R}_{v}(r, v)$ is done onto each true range-speed response with interpolated peak information. Range-scale modification and speed-scale modification sharpens the peaks with conservation of the peak location. The modifications in range and speed are equivalent. $\mathrm{K}$-scale modification with a sharpening factor $\beta$ gives:

$$
u(k)=a\left(\beta\left(k-k_{0}\right)\right)^{2}+b\left(\beta\left(k-k_{0}\right)\right)+c .
$$

An infinite sharpening factor $\beta$ gives the impulse delta response function where the peak is located to the peak position. This method is fast and can be applied in real-time applications. In order to smooth the impulse response, an additional Gaussian filter could be applied with a scale factor of half the grid resolution. This Gaussian filtering is a convolution and could be applied in real-time. Suppose $\hat{R}_{\text {gridded }}(r, v)$ is the gridded range-speed response and $G_{\sigma_{r}, \sigma_{v}}(r, v)$ the Gaussian filter with scale factor $\sigma_{r}$ in range and $\sigma_{v}$ in speed. The synthesis range-speed $\check{R}_{v}(r, v)$ response is:

$$
\check{R}_{v}(r, v)=\left(\hat{R}_{\text {gridded }} * G_{\sigma_{r}, \sigma_{v}}\right)(r, v)
$$

\section{E. Projections}

The vertical and horizontal range-speed-time amplitude $\check{R}_{v}(r, v, t), \check{R}_{h}(r, v, t)$ and aspect-angle $\alpha_{v}(r, v, t), \alpha_{h}(r, v, t)$ give the total feature space. Projection of the total feature space gives some insight in the human motion if for example tracking is possible. The projections are indicated with a $P$. The rangespeed response is calculated by integration over the time:

$$
P_{v}(r, v)=\sum_{\text {all } t} \check{R}_{v}(r, v, t)
$$

The angle-speed-time response is:

$$
P_{v}\left(\alpha_{v}, v, t\right)=\sum_{\text {all } r} \check{R}_{v}(r, v, t) \delta\left(\alpha_{v}-\alpha_{v}(r, v, t)\right)
$$

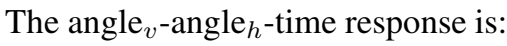

$$
\begin{aligned}
P\left(\alpha_{v}, \alpha_{h}, t\right)= & \sum_{\text {all } r \text { all } v} \sqrt{\check{R}_{v}(r, v, t) \check{R}_{h}(r, v, t)} \\
& \delta\left(\alpha_{v}-\alpha_{v}(r, v, t)\right) \delta\left(\alpha_{h}-\alpha_{h}(r, v, t)\right)
\end{aligned}
$$

This equation is the basis of radar-imaging where all responses are projected onto a plane with horizontal and vertical aspect angle.

\section{F. Kalman filtering}

Kalman filtering is applied to the peaks in the total feature space, see [2]. The peak parameters are interpolated range, interpolated speed, vertical observation angle, horizontal observation angle and amplitude. Three Kalman filters are implemented: first first filter tracks the range and speed, the second filter tracks the horizontal observation angle and the third filter tracks the vertical observation angle. One composite filter is not preferred due to the multipath observation angle errors. The horizontal angle and vertical observation angle have different multipath characteristics.

\section{RESULTS}

This section gives examples of the information extraction applied to real radar measurements. Two dual receiver channel interferometric FMCW radars where used. Figure 1 shows the measurement setup with the two radars.

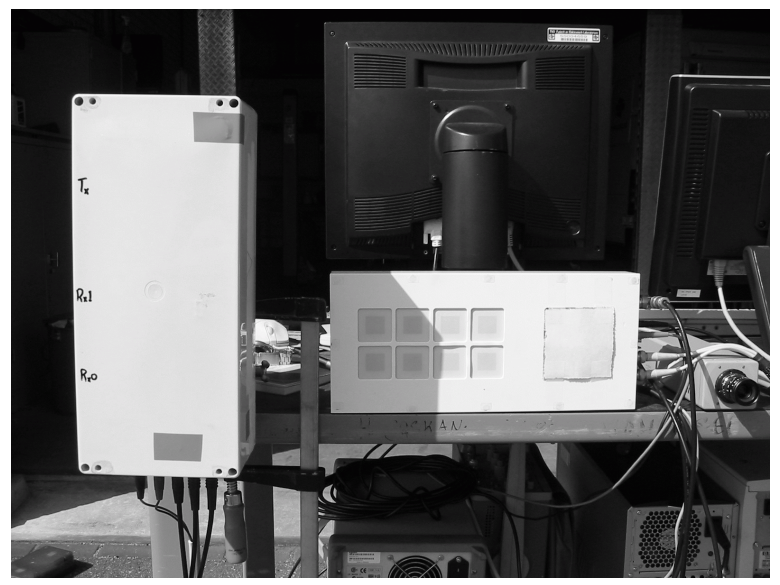

Fig. 1. Measurement setup with right the horizontal FMCW radar and left the vertical interferometric FMCW radar.

The carrier frequency of both radars is $5.7 \mathrm{GHz}$ with $\Delta R=$ $0.375 \mathrm{~m}, \Delta v=0.086 \mathrm{~m} / \mathrm{s}$ and $\Delta T=10 \mathrm{~ms}$ the update time between succeeding range-speed responses. Each radar has one transmitter patch antenna and two receiver path antennas. The beamwidth of both antennas is 22 degrees. The two radars transmit simultaneously in the same frequency band. To prevent interference between both transmitted signals, one transmitted radar signal has a delay. This delay is a fraction 
of the sweep time. A number of experiments have been done with static corner reflectors to evaluate the setup. Scenarios were evaluated with sitting humans, walking human, running human, cyclist and cars. The results of an outbound walking human are presented in this section. The outbound walking human has $15 \mathrm{~m}$ range and a speed of $1.5 \mathrm{~m} / \mathrm{s}$. The presented results are gated around the range of the human.

\section{A. Deconvolution}

The range-speed responses of nine range elements are shown in Figure 2. The walking human appears in the third range element of this figure and walks through successive range elements. The middle line is the zero speed, above negative speed and below positive speed. The thick response originates from the human body with $1.5 \mathrm{~m} / \mathrm{s}$ speed, the other responses originates from the human elements. One foot lifts up at $(0.3 \mathrm{~s}, 3.6 \mathrm{~m})$, put onto the ground at $(0.4 \mathrm{~s}, 4.4 \mathrm{~m})$, lift up at $(0.75 \mathrm{~s}, 4.7 \mathrm{~m})$, put onto the ground at $(0.9 \mathrm{~s}, 5.2 \mathrm{~m})$ and lift up at $(1.4 \mathrm{~s}, 5.2 \mathrm{~m})$. The other feet has asymmetric motion. The range-extent is 5 range elements. The negative speeds are ghost multipath responses of one leg with the other leg. Measurements of different observation angles show this behaviour. The human response with positive speed belongs to valid responses and multipath reponses. The ground reflections cause multipath ghosts which appears to originate below ground or above the valid observation angle of the response.
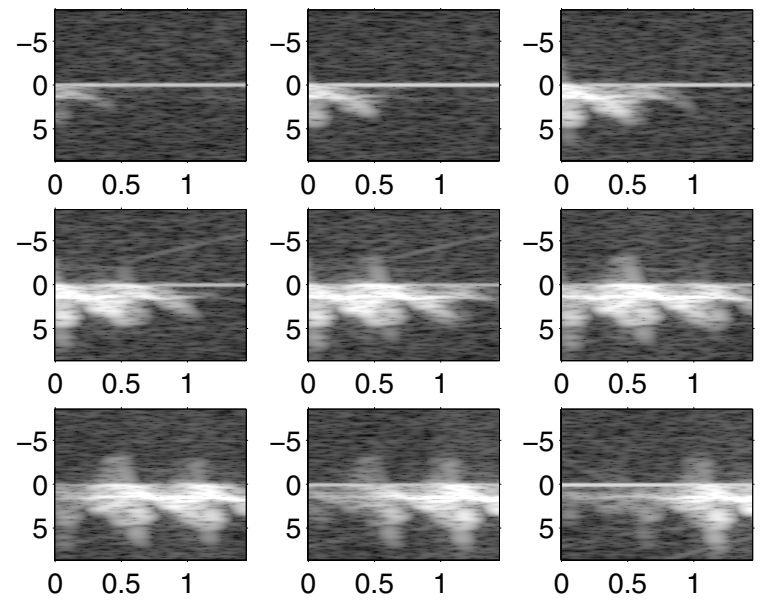

Fig. 2. Succeeding raw range-speed responses of a walking human in front of the vertical interferometric radar. Top left is the $\mathrm{n}$-th range element, top middle the $(\mathrm{n}+1)$-th range element, etc. The intensity is the received radar signal power.

The deconvolved range-speed is shown in 3. The rangeextent is less than 2 range elements. Note the significantly better clarity achieved in the deconvolved response. An artefact is the signal suppression around the peak positions.
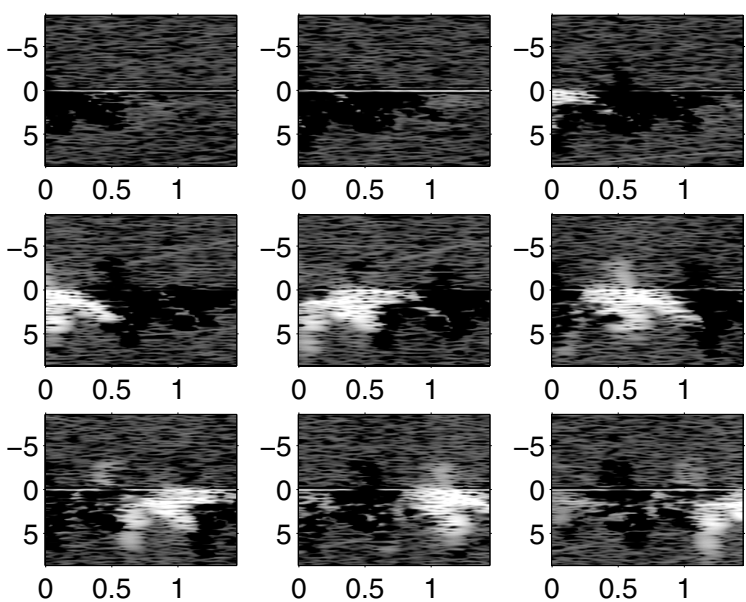

Fig. 3. Succeeding deconvolved range-speed responses of a walking human in front of the vertical interferometric radar. Top left is the $\mathrm{n}$-th range element, top middle the $(\mathrm{n}+1)$-th range elements, etc. The intensity is the received radar signal power.

\section{B. Peak Interpolation}

Figures 4, 5 and 6 show the interpolated peak positions in range-speed-time projections. The range positions give accurate ranges of the peaks. The interpolation gives no preference for specific ranges. The range positions lie on the diagonal starting at $(3.5 \mathrm{~m}, 0 \mathrm{~s})$ and ending at $(5.5 \mathrm{~m}, 1.5 \mathrm{~s})$. The speed positions are accurate and show constant speed preferences.

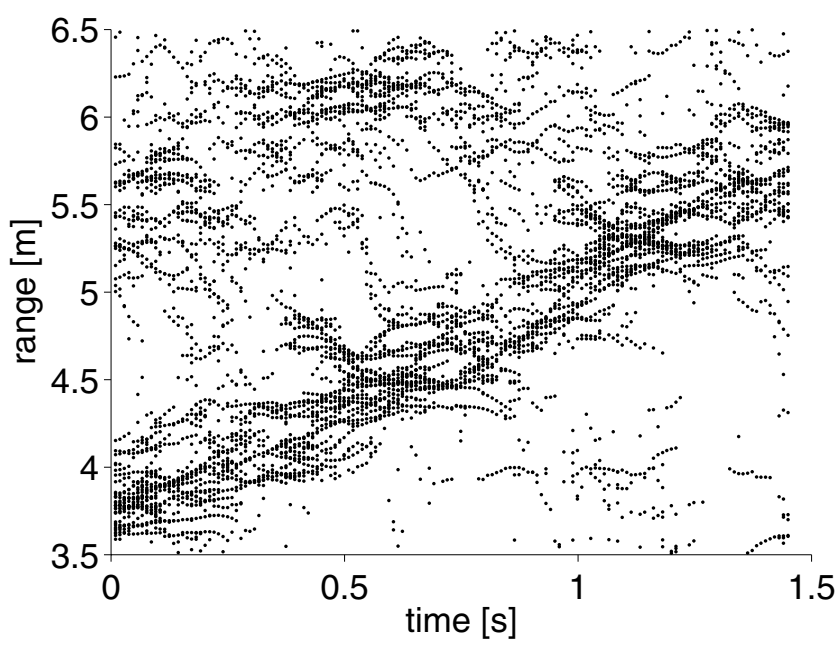

Fig. 4. Time-range response of a walking human in front of the vertical interferometric radar.

Sometimes the range position shows a speed and acceleration which differ from the estimated speed. In these cases the kinematics of the estimated range position differs with the kinematics of the estimated speed position. One explanation is 


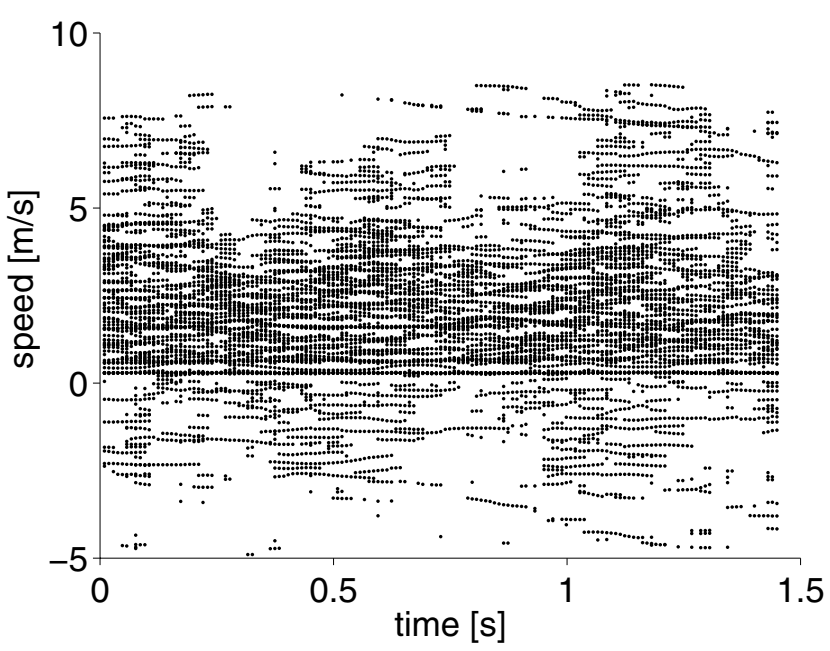

Fig. 5. Time-speed response of a walking human in front of the vertical interferometric radar.

the acceleration that disturbs the speed estimate more than the range estimate, the speed peak width caused by acceleration is greater than the range peak width. The range-speed response shows the contacts of the feet with the ground with positions $(3.6 \mathrm{~m}, 0 \mathrm{~m} / \mathrm{s}),(4.4 \mathrm{~m}, 0 \mathrm{~m} / \mathrm{s})$ and $(5.2 \mathrm{~m}, 0 \mathrm{~m} / \mathrm{s})$. The rangespeed response show no clear relation between range kinematics and speed kinematics.

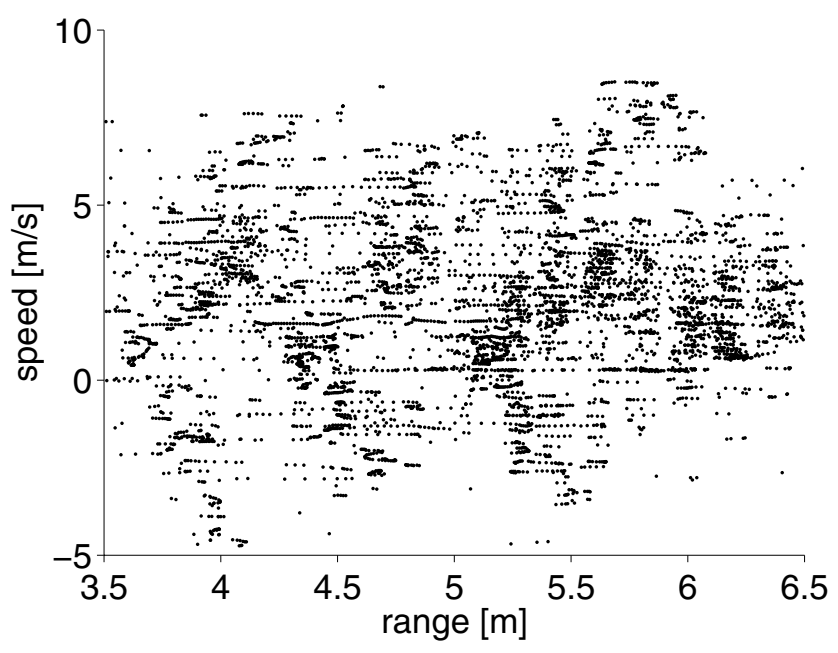

Fig. 6. Range-speed response of a walking human in front of the vertical interferometric radar.

\section{Tracking}

The results of the peak position tracking are split into only vertical radar tracking and combined vertical-horizontal radar tracking. The horizontal radar performs less than the vertical radar and causes less combined detection. The graphs in Figure
7 shows the vertical radar tracking. As mentioned above the tracks show constant speed preference and there is a mismatch between the range estimate and the speed estimate, the Kalman tracker gives the best of both. The vertical observation angle of the human response lie around -4 degrees. The responses of the human limbs lie around this value and show no multipath while the angles of the body response vary over all observation angles due to multipath. In spite of the multipath the angle Kalman filter tracks the peaks well.
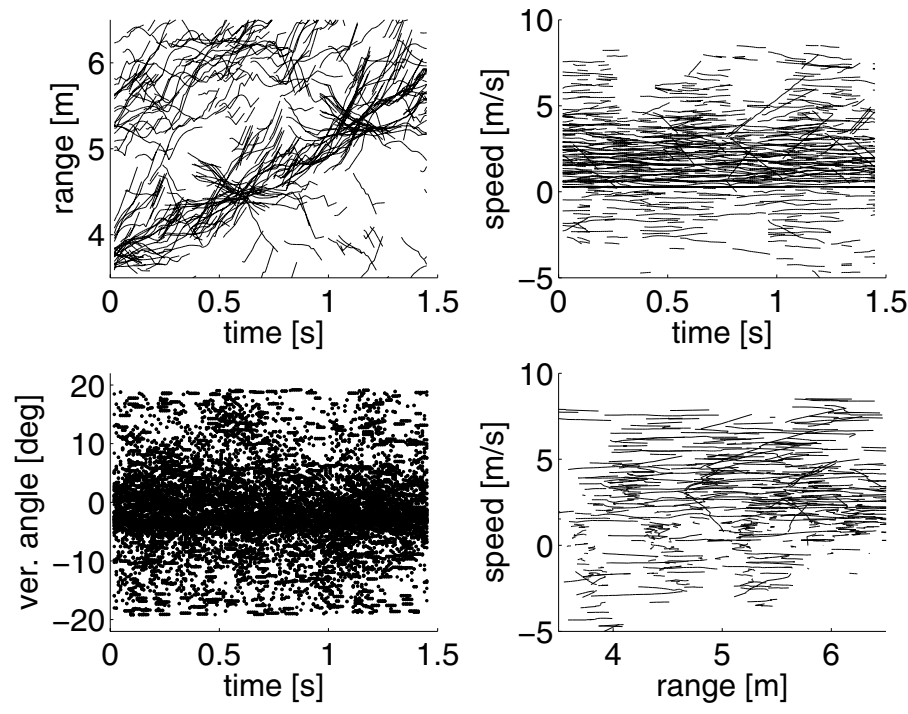

Fig. 7. Tracking results vertical radar. Left top time-range tracks, right top time-speed tracks, left bottom vertical observation angle tracks and right botton range-speed tracks.

The graphs in Figure 8 shows the combined verticalhorizontal radar tracking. The non-multipath responses lie around -4 degrees vertical and -4 degrees horizontal. The less combined detections give smaller number of tracks. The behaviour is equivalent with the vertical radar alone. 

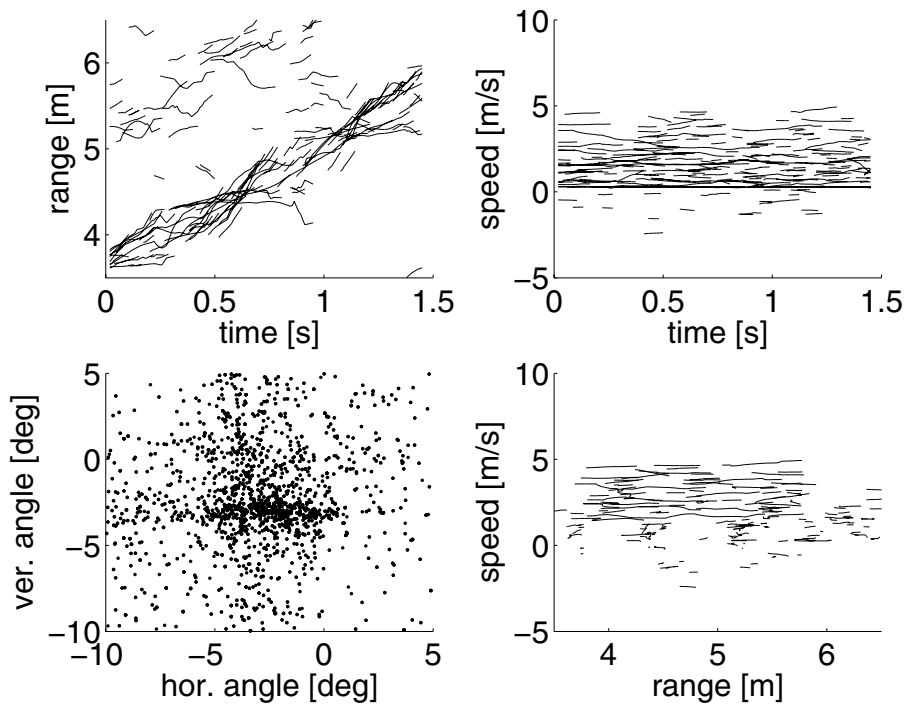

Fig. 8. Tracking results combined vertical and horizontal radar. Left top time-range tracks, right top time-speed tracks, left bottom vertical observation angle tracks and right botton range-speed tracks.

\section{CONCLUSIONS}

In this paper an analysis technique of range-speed radar sequences has been presented. We modelled the extended radar target with a sum of individual moving point scatterers in space. The technique first deconvolved the range-speed response to reveal the inner structure of extended targets. The second step estimate interpolated range-speed positions and, if available, observation angle information. A tracker combines different range-speed positions with observation angles. The point scatterer trajectories give the scattering points of the target.

It has been shown that the deconvolution is very successful in revealing the inner structure of an extended target and that range-speed interpolation gives accurate positions. On certain occasions the range estimate kinematics differ from the speed position kinematics. The Kalman filtering works correct and tracks also the multipath angle positions.

\section{REFERENCES}

[1] M. Abe and J.O. Smith. Am/fm rate estimation and bias correction for time-varying sinusoidal modeling. Technical Report Stan-M-118, Center for computer research in music and acoustics department of music, Stanford University, October 2004

[2] Y. Bar-Shalom and X.R. Li. Estimation and Tracking: Principles, Techniques, and Software. Artech House, 1993.

[3] V.C. Chen, R. Li, S.S. Ho, and H. Wechsler. Micro-doppler effect in radar phenomenon, model, and simulation study. IEEE Transactions on Aerospace and Electronic Systems, Volume 42(No. 1):pp 2-21, 2006.

[4] P. van Dorp and Groen F.C.A. Human walking estimation with radar. IEE Proc.-Radar Sonar Navigation., Volume 150(No. 5):pp 356-365, October 2003.
[5] P. van Dorp and Groen F.C.A. Real-time human walking estimation with radar. Proceedings International Radar Symposium (IRS) 2003, Dresden Germany, 2003.

[6] P van Dorp and F.C.A. Groen. Feature-based human motion parameter estimation with radar. To be published, 2007.

[7] D.J. Fleet and A.D. Jepson. Computation of component image velocity from local phase information. International Journal of Computer Vision, Vol. 5(No. 1):pp. 77-104, 1990.

[8] D.J. Fleet and K. Langley. Computational analysis of non-fourier motion. Vision Research, Vol. 34(No. 22):pp. 3057-3079, 1994.

[9] D.H. Hoekman and C. Varekamp. Observation of tropical rain forest trees by airborne high-resolution interferometricradar. ESA SP-489, pages 47-56, November 2000.

[10] H. Ling. Microdoppler exploitation: Preliminary data collection and analysis. NICOP Meeting London, June 72004.

[11] L.B. Lucy. An iterative technique for the rectification of observed distributions. The Astronomical Journal, Volume 79(Number 6):pp. 745-754, June 1974.

[12] L. Marple. Sharping techniques for sensor feature enhancement. May 26 2005

[13] A.K. Mitra, M. Kobold, T. Lewis, and R. Williams. Theoretical radar-doppler models for pivoting mechanical and biological objects-ofinterest. Proceeding SPIE, Algorithms for Synthetic Aperature Radar Imagery XIII, Volume 6237:25-32, May 2006.

[14] D.T. Sandwell and E.J. Price. Phase gradient approach to stacking interferograms. Journal of geophysical Research, Vol. 103(No. B12):pp. 30183-30204, 1998.

[15] M.I. Skolnik. Radar Handbook, volume Second Edition. Mc Graw Hill, 1990.

[16] J.O. Smith and X. Serra. Parshl: An analysis/synthesis program for non-harmonic sounds based on asinusoidalrepresentation. Technical Report CCRMA STAN-M-43, Center for Computer Research in Music and Acoustics (CCRMA), DepartmentofMusic, Standford University, Stanford, California 94305.

[17] T. Thayaparan, S. Abrol, and Riseborough. Micro-doppler radar signatures for intelligent target recognition. Technical Report DRDC Ottawa TM 2004-170, Defence Research and Development Canada - Ottawa, Ottawa, September 2004. 